PROCEEDINGS OF THE

AMERICAN MATHEMATICAL SOCIETY

Volume 127, Number 12, Pages 3475-3480

S 0002-9939(99)04944-8

Article electronically published on May 13, 1999

\title{
ON TIGHTNESS AND DEPTH IN SUPERATOMIC BOOLEAN ALGEBRAS
}

\author{
SAHARON SHELAH AND OTMAR SPINAS
}

(Communicated by Carl G. Jockusch, Jr.)

\begin{abstract}
We introduce a large cardinal property which is consistent with $L$ and show that for every superatomic Boolean algebra $B$ and every cardinal $\lambda$ with the large cardinal property, if tightness ${ }^{+}(B) \geq \lambda^{+}$, then $\operatorname{depth}(B) \geq \lambda$. This improves a theorem of Dow and Monk.
\end{abstract}

In [DM, Theorem C], Dow and Monk have shown that if $\lambda$ is a Ramsey cardinal (see [J, p.328]), then every superatomic Boolean algebra with tightness at least $\lambda^{+}$has depth at least $\lambda$. Recall that a Boolean algebra $B$ is superatomic iff every homomorphic image of $B$ is atomic. The depth of $B$ is the supremum of all cardinals $\lambda$ such that there is a sequence $\left(b_{\alpha}: \alpha<\lambda\right.$ ) in $B$ with $b_{\beta}<b_{\alpha}$ for all $\alpha<\beta<\lambda$ (a well-ordered chain of length $\lambda$ ). Then depth ${ }^{+}$of $B$ is the first cardinal $\lambda$ such that there is no well-ordered chain of length $\lambda$ in $B$. The tightness of $B$ is the supremum of all cardinals $\lambda$ such that $B$ has a free sequence of length $\lambda$, where a sequence $\left(b_{\alpha}: \alpha<\lambda\right)$ is called free provided that if $\Gamma$ and $\Delta$ are finite subsets of $\lambda$ such that $\alpha<\beta$ for all $\alpha \in \Gamma$ and $\beta \in \Delta$, then

$$
\bigcap_{\alpha \in \Gamma}-b_{\alpha} \cap \bigcap_{\beta \in \Delta} b_{\beta} \neq 0 .
$$

By tightness $^{+}(B)$ we denote the first cardinal $\lambda$ for which there is no free sequence of length $\lambda$ in $B$.

For $b \in B$ we sometimes write $b^{0}$ for $-b$ and $b^{1}$ for $b$.

We improve Theorem $\mathrm{C}$ from $[\mathrm{DM}]$ in two directions. We introduce a large cardinal property which is much weaker than Ramseyness and even consistent with $L$ (the constructible universe) and show that in Theorem $\mathrm{C}$ from [DM] it suffices to assume that $\lambda$ has this property. Moreover we show that it suffices to assume $\operatorname{tightness}^{+}(B) \geq \lambda^{+}$instead of tightness $(B) \geq \lambda^{+}$to conclude that $\operatorname{depth}(B) \geq \lambda$. In particular we get:

Theorem 1. Suppose that $0^{\sharp}$ exists. Let $B$ be a superatomic Boolean algebra in the constructible universe $L$, and let $\lambda$ be an uncountable cardinal in $V$. Then in $L$ it is true that tightness ${ }^{+}(B) \geq \lambda^{+}$implies that depth ${ }^{+}(B) \geq \lambda$.

Received by the editors November 18, 1997 and, in revised form, February 13, 1998.

1991 Mathematics Subject Classification. Primary 06E05.

The first author was supported by the Basic Research Foundation of the Israel Academy of Sciences; publication 663 .

The second author was partially supported by the Alexander von Humboldt Foundation and grant 2124-045702.95/1 of the Swiss National Science Foundation.

(C)1999 American Mathematical Society 
For the theory of $0^{\sharp}$ see $[\mathrm{J}, \S 30]$. Note that $\lambda$ as in Theorem 1 is a limit cardinal in $L$; hence it suffices to show that, in $L, \operatorname{depth}(B) \geq \kappa$ for all cardinals $\kappa<\lambda$. As was the case with the proof of Theorem $\mathrm{C}$ of $[\mathrm{DM}]$, we can't show that, under the assumptions of Theorem $1, \operatorname{depth}(B)=\lambda$ is attained, i.e. that there is a well-ordered chain of length $\lambda$.

For the proof we consider the following large cardinal property:

Definition 2. Let $\lambda, \kappa, \theta$ be infinite cardinals, and let $\gamma$ be an ordinal. The relation $R_{\gamma}(\lambda, \kappa, \theta)$ is defined as follows:

For every $c:[\lambda]^{<\omega} \rightarrow \theta$ there exists $A \subseteq \lambda$ of order-type $\gamma$, such that for every $u \in[A]^{<\omega}$ there exists $B \subseteq \lambda$ of order-type $\kappa$ such that $\forall w \in[B]^{|u|} \quad c(w)=c(u)$.

Lemma 3. Assume $R_{\gamma}(\lambda, \kappa, \theta)$, where $\gamma$ is a limit ordinal. For every $c:[\lambda]^{<\omega} \rightarrow \theta$ there exists $A \subseteq \lambda$ as in the definition of $R_{\gamma}(\lambda, \kappa, \theta)$ such that additionally $c \uparrow[A]^{n}$ is constant for every $n<\omega$.

Proof. Define $c^{\prime}$ on $[\lambda]^{<\omega}$ by

$$
c^{\prime}\left\{\beta_{0}, \ldots, \beta_{n-1}\right\}=\left\{\left(v, c\left\{\beta_{i}: i \in v\right\}\right): v \subseteq n\right\} .
$$

As $\theta$ is infinite we can easily code the values of $c^{\prime}$ as ordinals in $\theta$ and therefore apply $R_{\gamma}(\lambda, \kappa, \theta)$ to it. We get $A \subseteq \lambda$ of order-type $\gamma$. We shall prove that $c\left\lceil[A]^{n}\right.$ is constant, for every $n<\omega$. Fix $w_{1}, w_{2} \in[A]^{n}$. Since $\gamma$ is a limit, without loss of generality we may assume that $\max \left(w_{1}\right)<\min \left(w_{2}\right)$. Let $w=w_{1} \cup w_{2}$. By Definition 2 there exists $B \subseteq \lambda$, o.t. $B=\kappa$, such that $c^{\prime} \uparrow[B]^{2 n}$ is constant with value $c^{\prime}(w)$. Let $\left(\beta_{\nu}: \nu<\kappa\right)$ be the increasing enumeration of $B$. We have

$$
c^{\prime}\left\{\beta_{0}, \ldots, \beta_{2 n-1}\right\}=c^{\prime}\left\{\beta_{n}, \ldots, \beta_{3 n-1}\right\} .
$$

By the definition of $c^{\prime}$ we get

$$
c\left\{\beta_{0}, \ldots, \beta_{n-1}\right\}=c\left\{\beta_{n}, \ldots, \beta_{2 n-1}\right\}=: c_{0} .
$$

This information is coded in $c^{\prime}\left\{\beta_{0}, \ldots, \beta_{2 n-1}\right\}$, i.e.

$$
\left(\{0, \ldots, n-1\}, c_{0}\right),\left(\{n, \ldots, 2 n-1\}, c_{0}\right) \in c^{\prime}\left\{\beta_{0}, \ldots, \beta_{2 n-1}\right\} .
$$

As $c^{\prime}\left\{\beta_{0}, \ldots, \beta_{2 n-1}\right\}=c^{\prime}(w)$, we conclude $c\left(w_{1}\right)=c\left(w_{2}\right)=c_{0}$.

Theorem 4. Assume $R_{\gamma}(\lambda, \kappa, \omega)$, where $\gamma$ is a limit ordinal. If $B$ is a Boolean algebra and $\left(a_{\nu}: \nu<\lambda\right)$ is a sequence in $B$, then one of the following holds:

(a) there exists $A \subseteq \lambda$, o.t. $(A)=\gamma$, such that $\left(a_{\nu}: \nu \in A\right)$ is independent;

(b) there exist $n<\omega$ and a strictly increasing sequence $\left(\beta_{\nu}: \nu<\kappa\right)$ in $\lambda$ such that, letting

$$
b_{\nu}=\bigcup_{k<n} \bigcap_{l<n} a_{\beta_{n^{2} \nu+n k+l}},
$$

we have that $\left(b_{\nu}: \nu<\kappa\right)$ is constant;

(c) there exists a strictly decreasing sequence in $B$ of length $\kappa$.

Corollary 5. Assume $R_{\gamma}(\lambda, \kappa, \omega)$, where $\gamma$ is a limit ordinal. If $B$ is a superatomic Boolean algebra, then tightness ${ }^{+}(B)>\lambda$ implies Depth ${ }^{+}(B)>\kappa$. 
Proof of Corollary 5. Let $\left(a_{\nu}: \nu<\lambda\right)$ be a free sequence in $B$. As a superatomic Boolean algebra does not have an infinite independent subset, (a) is impossible. Suppose (b) were true. Define $b_{\nu}$ as in $(*)$. Clearly we have

$$
\begin{gathered}
-b_{\nu} \geq \bigcap_{k, l<n} a_{\beta_{n^{2} \nu+n k+l}^{0}}^{0}, \text { and } \\
b_{\nu} \geq \bigcap_{k, l<n} a_{\beta_{n^{2} \nu+n k+l}} .
\end{gathered}
$$

Hence if $\nu<\mu$ and $b_{\nu}=b_{\mu}$, we obtain

$$
0=-b_{\nu} \cap b_{\mu} \geq \bigcap_{k, l<n} a_{\beta_{n^{2} \nu+n k+l}^{0}}^{0} \cap \bigcap_{k, l<n} a_{\beta_{n^{2} \mu+n k+l}} .
$$

This contradicts freeness of $\left(a_{\nu}: \nu<\kappa\right)$. We conclude that (c) must hold.

Proof of Theorem 4. Define $c:[\lambda]^{<\omega} \rightarrow\left[{ }^{<\omega} 2\right]^{<\omega}$ by

$$
c\left\{\beta_{0}<\cdots<\beta_{n-1}\right\}=\left\{\eta \in{ }^{n} 2: \bigcap_{i<n} a_{\beta_{i}}^{\eta(i)}=0\right\} .
$$

Note that $c\left\{\beta_{0}<\cdots<\beta_{n-1}\right\}=c\left\{\alpha_{0}<\cdots<\alpha_{n-1}\right\}$ implies that $\left\{a_{\beta_{0}}, \ldots, a_{\beta_{n-1}}\right\}$ and $\left\{a_{\alpha_{0}}, \ldots, a_{\alpha_{n-1}}\right\}$ have the same quantifier-free diagram, i.e. for every quantifierfree formula $\phi\left(x_{0}, \ldots, x_{n-1}\right)$ in the language of Boolean algebra,

$$
B \models \phi\left[a_{\beta_{0}}, \ldots, a_{\beta_{n-1}}\right] \Leftrightarrow B \models \phi\left[a_{\alpha_{0}}, \ldots, a_{\alpha_{n-1}}\right] .
$$

Let $A \subseteq \lambda$ be as guaranteed for $c$ by $R_{\gamma}(\lambda, \kappa, \omega)$. By Lemma 3 we may assume that $c \uparrow[A]^{n}$ is constant, for every $n<\omega$.

If $\left(a_{\alpha}: \alpha \in A\right)$ is independent, we are done. Therefore we may assume that this is false. For $m<\omega$ define

$$
\Gamma_{m}=\left\{\eta \in{ }^{m} 2: \exists\left\{\beta_{0}<\cdots<\beta_{m-1}\right\} \subseteq A \bigcap_{i<m} a_{\beta_{i}}^{\eta(i)}=0\right\} .
$$

By assumption, in the definition of $\Gamma_{m}$ the existential quantifier can be replaced by a universal one to give the same set. There exists $m<\omega$ such that $\Gamma_{m} \neq \emptyset$. Define

$$
\Gamma_{m}^{\prime}=\left\{\eta \in \Gamma_{m}: \text { no proper subsequence of } \eta \text { belongs to } \bigcup_{k<m} \Gamma_{k}\right\} .
$$

By Kruscal's Theorem $[\mathrm{K}]$, we have that $\bigcup_{m<\omega} \Gamma_{m}^{\prime}$ is finite. Let $n^{*}$ be minimal such that $\bigcup_{m<\omega} \Gamma_{m}^{\prime}=\bigcup_{m<n^{*}} \Gamma_{m}^{\prime}$. Then clearly we have that, for every $m<\omega$ and $\eta \in \Gamma_{m}, \eta$ has a subsequence in $\bigcup_{k<n^{*}} \Gamma_{k}^{\prime}$. Let $m^{*}=\left(n^{*}\right)^{2}$, and let

$$
\tau\left(x_{0}, \ldots, x_{m^{*}-1}\right)=\bigcup_{l<n^{*}} \bigcap_{k<n^{*}} x_{n^{*} l+k} .
$$

Claim 1. If $\eta \in m^{*} 2, t \in\{0,1\}$, and $\tau\left[\eta(0), \ldots, \eta\left(m^{*}-1\right)\right]=t$ in the Boolean algebra $\{0,1\}$, then $\left|\left\{i<m^{*}: \eta(i)=t\right\}\right| \geq n^{*}$.

Let $\left(\beta_{\nu}: \nu<\gamma\right)$ be the strictly increasing enumeration of $A$, and define

$$
b_{\nu}=\tau\left[a_{\beta_{m^{*} \nu}}, a_{\beta_{m^{*} \nu+1}}, \ldots, a_{\beta_{m^{*} \nu+m^{*}-1}}\right],
$$

for every $\nu<\gamma$, where the evaluation of $\tau$ takes place in $B$, of course. It is easy to see that the sequence $\left(b_{\nu}: \nu<\gamma\right)$ inherits from $\left(a_{\beta_{\nu}}: \nu<\gamma\right)$ the property that any two finite subsequences of same length have the same quantifier-free diagram. 
Claim 2. If $\eta \in \Gamma_{n}$, then $\bigcap_{i<n} b_{i}^{\eta(i)}=0$.

Proof of Claim 2. Otherwise there exists an ultrafilter $D$ on $B$ such that $\bigcap_{i<n} b_{i}^{\eta(i)}$ $\in D$. Define $\zeta \in{ }^{n m^{*}} 2$ by $\zeta(i)=1$ iff $a_{\beta_{i}} \in D$. Then $\bigcap_{i<n m^{*}} a_{\beta_{i}}^{\zeta(i)} \in D$, and hence $\zeta \notin \Gamma_{n m^{*}}$. Let $h: B \rightarrow B / D=\{0,1\}$ be the canonical homomorphism induced by $D$. We calculate

$$
\begin{aligned}
1=h\left(\bigcap_{i<n} b_{i}^{\eta(i)}\right) & =\bigcap_{i<n} h\left(b_{i}\right)^{\eta(i)}=\bigcap_{i<n} \tau\left[h\left(a_{\beta_{m^{*} i}}\right), \ldots, h\left(a_{\beta_{m^{*}(i+1)-1}}\right)\right]^{\eta(i)} \\
& =\bigcap_{i<n} \tau\left[\zeta\left(m^{*} i\right), \ldots, \zeta\left(m^{*} i+k\right), \ldots, \zeta\left(m^{*}(i+1)-1\right)\right]^{\eta(i)} .
\end{aligned}
$$

We conclude that $\tau\left[\zeta\left(m^{*} i\right), \ldots, \zeta\left(m^{*} i+k\right), \ldots, \zeta\left(m^{*}(i+1)-1\right)\right]=\eta(i)$, for all $i<n$, and hence by Claim 1 we can choose $j_{i} \in\left[m^{*} i, m^{*}(i+1)\right)$ such that $\zeta\left(j_{i}\right)=\eta(i)$. Clearly $i_{0}<i_{1}$ implies that $j_{i_{0}}<j_{i_{1}}$. But this implies $\zeta \in \Gamma_{n m^{*}}$, a contradiction.

Claim 3. If $t<\omega, \eta \in \Gamma_{n}, 0=k_{0}<k_{1}<\cdots<k_{t}=n$, and $\eta \uparrow\left[k_{i}, k_{i+1}\right)$ is constant for all $i<t$, and if $\rho \in{ }^{t} 2$ is defined by $\rho(i)=\eta\left(k_{i}\right)$, then $\bigcap_{i<t} b_{i}^{\rho(i)}=0$.

Proof of Claim 3. Wlog we may assume that $\eta \in \Gamma_{n}^{\prime}$ for some $n<n^{*}$. Indeed, otherwise we can find $m<n^{*}, \eta^{\prime} \in \Gamma_{m}^{\prime}$ and some increasing $h: m \rightarrow n$ such that $\eta^{\prime}(i)=\eta(h(i))$, for all $i<m$. Then $\left\{h^{-1}\left[k_{i}, k_{i+1}\right): i<t\right\}$ equals $\left\{\left[l_{i}, l_{i+1}\right): i<s\right\}$ for some $l_{0}=0<l_{1}<\cdots<l_{s-1}=m$. Note that $\eta^{\prime} \uparrow\left[l_{i}, l_{i+1}\right)$ is constant, and letting $\rho^{\prime} \in{ }^{s} 2$ be defined by $\rho^{\prime}(i)=\eta^{\prime}\left(l_{i}\right)$, we have $\rho^{\prime}(i)=\rho(h(i))$. Hence $\bigcap_{i<s} b_{i}^{\rho^{\prime}(i)}=0$ implies $\bigcap_{i<t} b_{i}^{\rho(i)}=0$.

Therefore we assume $\eta \in \Gamma_{n}^{\prime}$, for some $n<n^{*}$. Suppose we had $\bigcap_{i<t} b_{i}^{\rho(i)}>0$. Let $D$ be an ultrafilter on $B$ containing $\bigcap_{i<t} b_{i}^{\rho(i)}$. Let $h: B \rightarrow B / D$ be the canonical homomorphism. Define $\zeta \in{ }^{t m^{*}} 2$ such that $\zeta(i)=1$ iff $a_{i} \in D$. Hence $\zeta \notin \Gamma_{t m^{*}}$. We get

$$
h\left(\bigcap_{i<t} b_{i}^{\rho(i)}\right)=\bigcap_{i<t} \tau\left[\zeta\left(i m^{*}\right), \ldots, \zeta\left((i+1) m^{*}-1\right)\right]^{\rho(i)}=1 .
$$

Hence by Claim 1,

$$
\forall i<t \exists a_{i} \in\left[\left\{i m^{*}, \ldots,(i+1) m^{*}-1\right\}\right]^{n^{*}} \forall j \in a_{i} \quad \zeta(j)=\rho(i) .
$$

Define $\mu \in{ }^{t n^{*}} 2$ by $\mu(j)=\rho(i)$ iff $j \in\left[i n^{*},(i+1) n^{*}\right)$. Then $\mu$ is a subsequence of $\zeta$ and therefore $\mu \notin \Gamma_{t n^{*}}$. But also $\eta$ is a subsequence of $\mu$, and hence $\eta \notin \Gamma_{n}$, a contradiction.

Claim 4. Suppose $\rho \in{ }^{t} 2$ and $\bigcap_{i<t} b_{i}^{\rho(i)}=0$. Let $\zeta \in{ }^{m^{*}}{ }^{2}$ be defined such that $\zeta\left(m^{*} i\right)=\rho(i)$ and $\zeta\left\lceil\left[m^{*} i, m^{*}(i+1)\right)\right.$ is constant for every $i<t$. Then $\zeta \in \Gamma_{m^{*} t}$.

Proof of Claim 4. Otherwise, $\bigcap_{i<m^{*} t} a_{i}^{\zeta(i)}>0$. Let $D$ be an ultrafilter containing $\bigcap_{i<m^{*} t} a_{i}^{\zeta(i)}$. Let $h: B \rightarrow B / D$ be the canonical homomorphism. We have

$$
h\left(\bigcap_{i<t} b_{i}^{\rho(i)}\right)=\bigcap_{i<t} \tau\left[\zeta\left(m^{*} i\right), \ldots, \zeta\left(m^{*}(i+1)-1\right)\right]^{\rho(i)}=\bigcap_{i<t} \tau[\rho(i), \ldots, \rho(i)]^{\rho(i)}=1 .
$$

This is a contradiction.

Since we assume that $\left(a_{\alpha}: \alpha \in A\right)$ is not independent, by Claim 2 we can find $k^{*}<\omega$ minimal such that for some $\rho^{*} \in{ }^{*} 2, \bigcap_{i<k^{*}} b_{i}^{\rho^{*}(i)}=0$. Note that $\rho^{*}(i+1) \neq \rho^{*}(i)$ for every $i<k^{*}-1$. Indeed, otherwise let $\zeta \in \epsilon^{m^{*} k^{*}} 2$ be defined 
as in Claim 4. So $\zeta \in \Gamma_{m^{*} k^{*}}$. By Claim 3 we can find $\rho^{\prime}$ of shorter length than $\rho^{*}$ such that $\bigcap_{i<\left|\rho^{\prime}\right|} b_{i}^{\rho^{\prime}(i)}=0$, contradicting the minimal choice of $k^{*}$.

Suppose first that $k^{*}=1$. We conclude that $\left(b_{\nu}: \nu<\gamma\right)$ either is constantly 1 or 0 . The main part of the definition of $R_{\gamma}(\lambda, \kappa, \omega)$ then gives a sequence of length $\kappa$ as desired in (b) of Theorem 4.

Second suppose $k^{*}>1$. If $\bigcap_{i<k^{*}-2} b_{i}^{\rho^{*}(i)} \cap b_{k^{*}-2} \cap b_{k^{*}-1}^{0}=0$ and $\bigcap_{i<k^{*}-2} b_{i}^{\rho^{*}(i)} \cap$ $b_{k^{*}-2}^{0} \cap b_{k^{*}-1}=0$, then $\bigcap_{i<k^{*}-2} b_{i}^{\rho^{*}(i)} \cap b_{k^{*}-2}=\bigcap_{i<k^{*}-2} b_{i}^{\rho^{*}(i)} \cap b_{k^{*}-1}$, and an application of the main part of the definition of $R_{\gamma}(\lambda, \kappa, \omega)$ gives a sequence as desired in (b).

Otherwise, if $\rho^{*}\left(k^{*}-2\right)=1$ and $\rho^{*}\left(k^{*}-1\right)=0$, then

$$
\bigcap_{i<k^{*}-2} b_{i}^{\rho^{*}(i)} \cap b_{k^{*}-2}<\bigcap_{i<k^{*}-2} b_{i}^{\rho^{*}(i)} \cap b_{k^{*}-1},
$$

and applying the definition gives (c). A similar argument applies if $\rho^{*}\left(k^{*}-2\right)=0$ and $\rho^{*}\left(k^{*}-1\right)=1$.

Theorem 6. Assume the following:

(1) $0^{\sharp}$ exists,

(2) $V \models \lambda$ is an uncountable cardinal,

(3) $\kappa, \theta<\lambda$, and $L \models \kappa$ is a regular cardinal.

Then $L \models R_{\omega}(\lambda, \kappa, \theta)$.

Proof. Let $c:[\lambda]^{<\omega} \rightarrow \theta, c \in L$, be arbitrary.

Let $Y$ be the set of all $w \in[\lambda]^{<\omega}$ such that for every $n \leq|w|$ and $u \in[w]^{n}$ there exists $B \subseteq \lambda$ of order-type $\kappa$ in $L$ such that $\forall v \in[B]^{n} \quad c(u)=c(v)$. Clearly $Y \in L$.

Claim 1. If in $V$ there exists $A \in[\lambda]^{\omega}$ with $[A]^{<\omega} \subseteq Y$, then $L \models R_{\omega}(\lambda, \kappa, \theta)$.

Proof of Claim 1. Let $T$ be the set of all one-to-one sequences $\rho \in{ }^{<\omega} \lambda$ with $\operatorname{ran}(\rho) \in Y$, ordered by extension. Then $T$ is a tree and by assumption, $T$ has an $\omega$-branch in $V$. By absoluteness, $T$ has an $\omega$-branch $b$ in $L$. Then $\operatorname{ran}(b)$ (or some subset) witnesses $L \models R_{\omega}(\lambda, \kappa, \theta)$.

Let $\left(i_{\nu}: \nu<\lambda^{+}\right)$be the increasing enumeration of the club of indiscernibles of $L_{\lambda^{+}}$. Then $\left(i_{\nu}: \nu<\lambda\right)$ is the club of indiscernibles of $L_{\lambda}$. As $c \in L_{\lambda^{+}}$there exist ordinals $\xi_{0}<\cdots<\xi_{p-1}<\lambda \leq \xi_{p}<\cdots<\xi_{q-1}<\lambda^{+}$and a Skolem term $t_{c}$ such that

$$
L_{\lambda^{+}}=c=t_{c}\left[i_{\xi_{0}}, \ldots, i_{\xi_{q-1}}\right] .
$$

By indiscernibility and remarkability (see [J, p.345]) it easily follows that if $\alpha^{*}=$ $\max \left\{\xi_{p-1}, \theta\right\}+1$, then $c \uparrow\left[\left\{i_{\nu}: \alpha^{*} \leq \nu<\lambda\right\}\right]^{n}$ is constant for every $n<\omega$, say with value $c_{n}$. Let $n<\omega$ be arbitrary. Let $\delta_{0}=i_{\alpha^{*}+\kappa}, \delta_{1}=i_{\alpha^{*}+\kappa+1}, \ldots, \delta_{n-1}=$ $i_{\alpha^{*}+\kappa+n-1}$.

Claim 2. For every $\alpha<\delta_{0}$ there exists a limit $\delta, \alpha<\delta<\delta_{0}$, such that for all $\beta_{0}<\cdots<\beta_{n-2}<\delta$ the following hold:

$(*)_{0} c\left\{\delta, \delta_{1}, \ldots, \delta_{n-1}\right\}=c\left\{\delta_{0}, \ldots, \delta_{n-1}\right\}\left(=c_{n}\right)$,

$(*)_{1} c\left\{\beta_{0}, \delta, \delta_{2}, \ldots, \delta_{n-1}\right\}=c\left\{\beta_{0}, \delta_{1}, \ldots, \delta_{n-1}\right\}$,

$(*)_{2} c\left\{\beta_{0}, \beta_{1}, \delta, \delta_{3}, \ldots, \delta_{n-1}\right\}=c\left\{\beta_{0}, \beta_{1}, \delta_{2}, \ldots, \delta_{n-1}\right\}$,

...

$(*)_{n-1} c\left\{\beta_{0}, \ldots, \beta_{n-2}, \delta\right\}=c\left\{\beta_{0}, \ldots, \beta_{n-2}, \delta_{n-1}\right\}$. 
Proof of Claim 2. Let $\alpha<\delta_{0}$ be arbitrary. Choose $\gamma<\kappa$ such that $\gamma$ is a limit and $i_{\alpha^{*}+\gamma}>\alpha$, and let $\delta=i_{\alpha^{*}+\gamma}$.

Then clearly $(*)_{0}$ holds.

In order to prove $(*)_{1}$, let $\beta<\delta$ be arbitrary. There exist ordinals $\nu_{0}<\cdots<$ $\nu_{k-1}<\alpha^{*}+\gamma$ and a Skolem term $t_{\beta}$ such that

$$
t_{\beta}^{L_{\lambda}}\left[i_{\nu_{0}}, \ldots, i_{\nu_{k-1}}\right]=\beta .
$$

Moreover there exist ordinals $\mu_{0}<\cdots<\mu_{l-1}<\alpha^{*}$ and a Skolem term $t$ such that (+) $\quad L_{\lambda^{+}} \models t\left[i_{\mu_{0}}, \ldots, i_{\mu_{l-1}}\right]=t_{c}\left[i_{\xi_{0}}, \ldots, i_{\xi_{q-1}}\right]\left\{t_{\beta}\left[i_{\nu_{0}}, \ldots, i_{\nu_{k-1}}\right], \delta_{1}, \ldots, \delta_{n-1}\right\}$.

Note that all indices of occurring indiscernibles, except for $\delta_{1}, \ldots, \delta_{n-1}$, either are at least $\lambda$ or else below $\alpha^{*}+\gamma$. We conclude that, in $(+), \delta_{1}$ can be replaced by $\delta$. The resulting statement is

$$
c\left\{\beta, \delta_{1}, \ldots, \delta_{n-1}\right\}=c\left\{\beta, \delta, \delta_{2}, \ldots, \delta_{n-1}\right\},
$$

as desired.

The proof of $(*)_{2}-(*)_{n-1}$ is similar.

It is clear that the statement of Claim 2 is absolute. Hence it is also true in $L$. Using this we shall prove that $\left[\left\{i_{\nu}: \alpha^{*} \leq \nu<\lambda\right\}\right]^{<\omega} \subseteq Y$. By Claim 1, this will suffice. We only have to prove that for every $n<\omega$ there exists $B \subseteq \lambda$ of order-type $\kappa$ such that $B \in L$ and $\forall v \in[B]^{n} \quad c(v)=c_{n}$. Fix $n<\omega$. Working in $L$, we construct $B$ inductively as $\left\{\gamma_{\nu}: \nu<\kappa\right\}$.

Fix $\delta_{0}<\delta_{1}<\cdots<\delta_{n-2}<\lambda$ as above. Apply Claim 2 in $L$ with $\alpha=0$ and obtain $\gamma_{0} \in\left(0, \delta_{0}\right)$. Suppose we have gotten $\left(\gamma_{\nu}: \nu<\mu\right)$ for some $\mu<\kappa$. Let $\gamma^{*}=\sup _{\nu<\mu} \gamma_{\nu}+1$. Since $\operatorname{cf}^{L}\left(\delta_{0}\right) \geq \kappa$ and $\left(\gamma_{\nu}: \nu<\mu\right) \in L$, we have that $\gamma^{*}<\delta_{0}$. Apply Claim 2 with $\alpha=\gamma^{*}$ and get $\gamma_{\mu} \in\left(\gamma^{*}, \delta_{0}\right)$.

We claim that $\left(\gamma_{\nu}: \nu<\kappa\right)$ is as desired. Indeed, let $\left\{\gamma_{\nu_{0}}<\gamma_{\nu_{1}}<\cdots<\gamma_{\nu_{n-1}}\right\}$ be arbitrary. We have

$$
\begin{aligned}
c\left\{\gamma_{\nu_{0}}, \ldots, \gamma_{\nu_{n-1}}\right\} & ={ }^{(*)_{n-1}} c\left\{\gamma_{\nu_{0}}, \ldots, \gamma_{\nu_{n-2}}, \delta_{n-1}\right\} \\
& ={ }^{(*)_{n-2}} c\left\{\gamma_{\nu_{0}}, \ldots, \gamma_{\nu_{n-3}}, \delta_{n-2}, \delta_{n-1}\right\} \\
& =\ldots \\
& ={ }^{(*)_{1}} c\left\{\gamma_{\nu_{0}}, \delta_{1}, \ldots, \delta_{n-1}\right\} \\
& ={ }^{(*)_{0}} c_{n} .
\end{aligned}
$$

\section{REFERENCES}

[DM] A. Dow and D. Monk, Depth, $\pi$-character, and tightness in superatomic Boolean algebras, Top. and its Appl. 75(1997), 183-199. MR 98a:54002

[J] T. Jech, Set Theory, Academic Press, New York, 1978. MR 80a:03062

$[\mathrm{K}] \quad$ J. Kruskal, Well-quasi ordering, the tree theorem and Vazsonyi's conjecture, Trans. Am. Math. Soc. 95(1960), 210-225. MR 22:2566

Institute of Mathematics, Hebrew University, Givat Ram, 91904 Jerusalem, Israel

E-mail address: shelah@math.huji.ac.il

Mathematik, ETH-Zentrum, 8092 Zürich, SwitZerland

E-mail address: spinas@math.ethz.ch 